CESIS Electronic Working Paper Series

Paper No. 201

\title{
Total Factor Productivity of Korean Manufacturing Industries: Comparison of Competing Models with Firm-Level Data
}

Donghyun Oha, Almas Heshmatib, and Hans Lööfa

October 2009 


\title{
Total Factor Productivity of Korean Manufacturing Industries: Comparison of Competing Models with Firm-Level Data
}

\author{
Donghyun $\mathrm{Oh}^{\mathrm{a} 1}$, Almas Heshmati ${ }^{\mathrm{b} 2}$, Hans Lööf ${ }^{\mathrm{a} 3}$ \\ a: Center of Excellence for Science and Innovation Studies, \\ Royal Institute of Technology, \\ b: Technology Management, Economics and Policy Program, \\ Seoul National University, Seoul, Korea.
}

October 12, 2009

\begin{abstract}
This paper presents the parametric estimation of the rates of technical change and total factor productivity (TFP) growth of 7,462 Korean manufacturing firms for the period 1987 to 2007. Two alternative formulations of technical change measured by the time trend and the general index approaches are estimated with panel data models assuming flexible functional forms. Several extensions of each approach are also considered and their benefits and limitations are discussed. In addition to making estimates of the TFP growth and its decomposition, the paper compares the parametric TFP growth measure with the non-parametric Solow residual serving as a benchmark. Several hypotheses related to technology level, firm sizes, industrial sectors, skill biased technological change and macroeconomic and industrial policies are tested to explain the growth patterns and heterogeneity in technical change, input biases and TFP growth rates. Using second regression analysis, the paper explores the determinants of TFP growth and their policy implications.
\end{abstract}

Key words: Total factor productivity; technical change; manufacturing industry; determinants of growth.

JEL classification: C23, C51, D24, L25, L60

\footnotetext{
${ }^{1}$ Corresponding author, e-mail: donghoh@infra.kth.se

${ }^{2}$ heshmati@snu.ac.kr

${ }^{3}$ hans1@infra.kth.se
} 


\section{Introduction}

Dynamic modeling of production functions has long been regarded as one of the interesting research topics by theoretical as well as applied researchers. The reason for its popularity is that finding appropriate production functions plays an important role in analyzing total factor productivity (TFP) growth and its decomposed sources. If a rich set of panel data is available, more sophisticated modeling can be conducted, enabling applied researchers to provide more reliable and practicable policy implications with respect to TFP growth. Considerable effort has been devoted to quantifying the rate of TFP growth and its components, and the following four main methodological strands have resulted: (a) econometric estimation of cost and production functions, (b) Divisia indexes, (c) exact index numbers and (d) nonparametric methods using linear programming (Diewert, 1981).

The econometric approach, which has dominated the applied research in the field of industrial economics, often assumes that technical change is generally represented by a simple time trend. It has a strong point in that it reveals long-run trends of technical change in an appropriate way when examining the behavior of manufacturing industries. This strong point comes from the fact that capital equipment, which rarely shows abrupt change over time, is the main determinant of long-run technical change and productivity growth. However, the use of the time trend model has been criticized for being merely the reflection of our ignorance about technical change. This weakness of the time trend model is overcome by the seminal work by Baltagi and Griffin (1988), in which the time trend is substituted by a general index in order to depict the unknown state of technology. The advantages of the general index model over the standard time trend model are summarized in Baltagi and Griffin (1988). The main advantage of the general index model is that it does not require any assumptions on the behavior of technical change.

We use parametric approaches to measuring TFP growth, technical change, returns to scale, biases in technical change and input elasticities of Korean manufacturing industries. The aforementioned two main strands of production functions, the time trend model (hereafter, TT1 model) and the general index model (hereafter, GI1 model), are used as a starting point of our model specifications in capturing the patterns of technical change. We also extend the TT1 and GI1 models since the basic models fail to provide firm-specific measures of technical change. The failure arises when: (a) the TT1 and GI1 models cease to provide firm-specific technical change if technical change is neutral, (b) firms confronting the same inputs and output prices yield the same measures of TFP growth, technical change, and returns to scale, for instance. In this sense, the TT1 and GI1 models play no role in measuring firm-specific TFP growth, technical change, returns to scale and biases insofar as one of the above conditions arises. Only intercepts are firm-specific with these specifications, which might not be sufficient to capture the economically meaningful firm-specific heterogeneity. Hence, it is necessary to alleviate the implicitly restrictive assumptions imposed on the conventional basic approaches. To consider this alleviation in our dynamic modeling, we allow flexibility by using less restrictive patterns in technical change. 
In order to examine TFP growth and its relevant measures with these alleviated assumptions and to provide more economically meaningful concepts inherent in the measures, we have extended the two basic models. The first extension of the TT1 model incorporates firm-specific technical change, and is labeled as the TT2 model, which adopts the Cornwell et al. (1990) model, where the time-varying technical inefficiency of the Cornwell et al. (1990) model is interpreted as the firm-specific neutral rate of technical change. The second extension of the TT1 model, the TT3 model, removes inherited restrictions further, by making all components firm-specific. All the interaction terms of time and input factors are set to be firm-specific in the TT3 model. The corresponding two extensions of the GI1 models are as follows. The GI2 model interprets the time-varying firm-specific technical inefficiency of the Lee and Schmidt (1993) model as neutral firm- and time-specific technical change. The GI3 model generalizes the GI2 model by allowing both neutral and non-neutral components of technical change to be firm- and time-specific. The parametric TFP growth measures are also further compared with the non-parametric Solow residuals. The latter serves as a benchmark.

This paper employs the aforementioned six models to investigate the patterns in TFP growth of Korean manufacturing industries for the 'roller-coaster period' of 1987-2007. The following are the reasons for choosing the study period. After the Korean War in 1950, Korea showed a very rapid economic growth due to state-led economic planning during the 1960s and 1970s. The manufacturing industries have been chosen to be the main engines for developing the economy ever since this period. In the 1980s, right after the assassination of General Park Jeong Hee, most of the state-led economic planning was challenged. This challenge was regarded as an attempt to remove the old regime and to replace it with a new one, although the latter was autocratic from the political point of view. High-ranked bureaucrats attempted to transform the economy into a freer market system with export-driven and conglomerate-friendly policies (Lee, 2002).

The Korean economy continued to grow until 1997, and reached per capita GDP of $\$ 10,000$ in that year. However, the economy encountered the Monetary Crisis in November 1997. Macroeconomic statistics show that the economy was affected severely by the Crisis. GDP decreased by 6.7 percent in 1998 and fixed investment contracted by almost 40 percent. Average monthly bankruptcies surpassed 3,000 in 1998. However, the economy recovered shortly after, and the government declared that the Crisis was formally ended in 2001. Despite this quick recovery from the 1997 crisis, another Crisis in 2003, the Credit Crunch Crisis, emerged and was overcome shortly after in the same year. The Credit Crunch Crisis was somewhat different from the Monetary Crisis in that the former was initiated by the high debts of households while the latter was initiated by the poor capital structure of enterprises.

Unlike previous studies, many of which employ macroeconomic tools to investigate the 'roller-coaster period', we examine this period from the microeconomic perspective. This attempt was justified for the following reasons. The microeconomic investigation, as a substitute of the macroeconomic approach, is likely to yield unexplored information about the crises. The rationale of this counterpart study is that the total sum of the behavior of micro agents is not necessarily the same as the aggregate macroeconomic output (Dopfer et al., 2004). We employ the aforementioned six econometric models in 
investigating firm-level TFP growth and its component during the roller-coaster period. We also use a large number of observations to guarantee robust and informative empirical investigation results.

The number of unique firms in our sample is 7,462 and the total number of observations is 60,868. A comparison is made of the measures of TFP growth and rate of technical change in the manufacturing industry. We also compare the scale properties of the industry regarding input elasticities, returns to scale, and input and scale biases calculated from the competing models. The determinants of TFP growth and their impacts are also investigated.

The rest of the paper is organized as follows. Data on the Korean manufacturing industry is presented in Section 2. Section 3 provides the theoretical framework of modeling TFP growth, technical change, input elasticities, returns to scale, and input and scale biases. Section 4 discusses the model specifications, estimation methods, specification tests and empirical results. Finally, Section 5 briefly concludes this paper.

\section{Data}

The data used in this study covers the period 1987-2007. The administrative data has been retrieved from the Korea Information Service (KIS-VALUE). The initial data set consists of 180,159 observations on 8,579 audited manufacturing firms in Korea.. Observations containing missing values in both the fixed assets and the value added have been removed. Then, missing values in fixed assets, value-added and number of employees were imputed by employing the bootstrapping imputation approach suggested by Little and An (2004).

Three variables are used in the empirical examination of the production function and computation of TFP growth. The value-added of each firm is used as a measure of output $(Y){ }^{i}$ Capital stock and labor $(\mathrm{K}$ and $\mathrm{L})$ are used as input variables. Fixed assets are used as a proxy for capital stock following Fu et al. (2008). We use the number of workers as a measure of labor input. Value-added and capital are deflated by using the consumer price index with 2000 as a base year. All the variables are transformed to logarithmic form before estimating the production function.

All the firms are grouped according to their size and technology levels to investigate their productivity growth according to these firm characteristics. Firms are categorized into four size classes based on the number of employees: fewer than 10, 10 to 50, 50 to 300 , and over 300 employees. The criteria for the technology levels are obtained from the OECD (2003). Every firm is categorized into one of four technology levels: high tech, high-medium tech, medium-low tech and low tech groups. 
Table 1. Descriptive statistics of key variables used in this study

\begin{tabular}{crrrrr}
\hline & Mean & StdDev & Median & Maximum & Minimum \\
\hline Value added (billion KRW) & $19,999.6$ & $158,130.2$ & $4,158.9$ & $13,575,657.1$ & 0.4 \\
Capital stock (billion KRW) & $51,768.1$ & $419,084.9$ & $6,502.8$ & $23,510,943.3$ & 3.0 \\
Number of employees & 316.8 & $1,471.0$ & 98.0 & $59,019.0$ & 1.0 \\
\hline
\end{tabular}

Table 1 provides summary statistics including the means, medians and standard deviations for the input and output variables used in this study. The fact that all the variables have mean values larger than the median indicates that the distributions of all the variables are skewed to the right. This means that a large number of firms have operated with small inputs and small output levels, and relatively few firms have operated with large inputs and output levels. The skewness of value added, capital and labor variables are 42.0, 27.6 and 21.6, respectively.

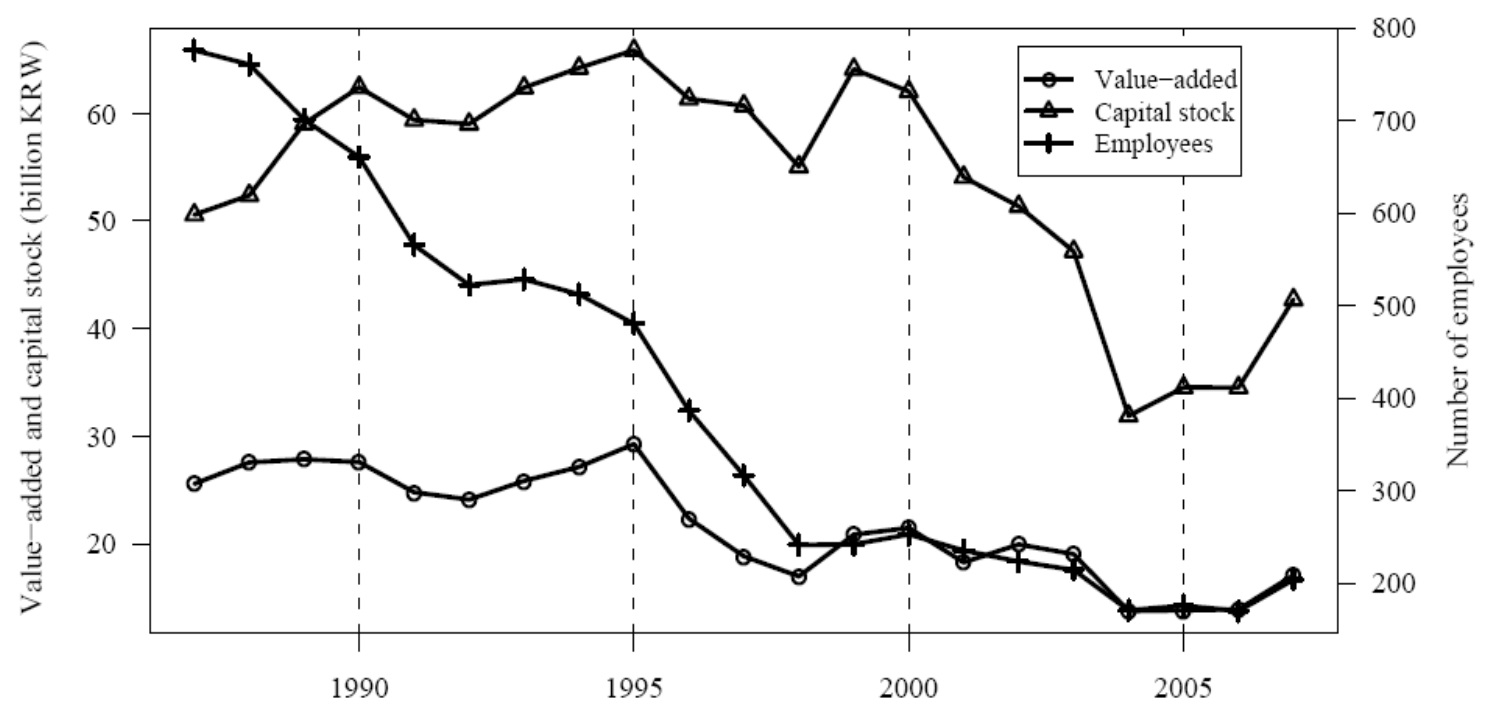

Figure 1. Development of average values of inputs and output.

Figure 1 shows the development of the mean values of value added, capital and employees over time. The value added increased on the whole during 1987-1995, whereas it shows a decreasing trend afterwards. The decreasing trend in average value added from 1995 indicates that the value added had started to decrease two years before the Monetary Crisis. A huge drop in the average value added can also be found for 2003. As already discussed, the economy suffered from the Credit Crunch Crisis in this year, as a result of which the affordability of consumers shrank substantially. The average number of employees constantly decreased during the study period. It is also shown that the average number of employees and capital dramatically decreased from 1995, indicating that the input factor market had already been affected two years before the 
Monetary Crisis. In 2003, a huge drop of input factors may be noted. In summary, markets for output and inputs had already been affected two years before the Monetary Crisis, whereas all the inputs and output were affected by the Credit Crunch Crisis at the same time as the crisis emerged. From these trends, one would expect a rather large fluctuation in the TFP growth of the Korean manufacturing industries, in particular during the two crises.

Table 2. Descriptive statistics of inputs and output by size and technology level of firms

\begin{tabular}{|c|c|c|c|c|c|c|c|c|c|}
\hline & \multicolumn{2}{|c|}{$\mathrm{Y}$ (Bil. KRW) } & \multicolumn{2}{|c|}{$\mathrm{K}$ (Bil. KRW) } & \multicolumn{2}{|c|}{$\mathrm{L}$} & \multirow{2}{*}{$\begin{array}{l}\text { Number } \\
\text { of obs. }\end{array}$} & \multirow{2}{*}{$\begin{array}{l}\text { Percent } \\
(\%)\end{array}$} & \multirow{2}{*}{$\begin{array}{c}\text { Cum. Perc. } \\
(\%)\end{array}$} \\
\hline & Mean & S.D. & Mean & S.D. & Mean & S.D. & & & \\
\hline \multicolumn{10}{|l|}{ By size } \\
\hline micro & 608.1 & 951.3 & 1746.6 & 3959.2 & 6.5 & 1.9 & 709 & 1.2 & 1.2 \\
\hline small & 1847.8 & 6428.3 & 3354.6 & 4498.9 & 31.4 & 11.0 & 15357 & 25.2 & 26.4 \\
\hline medium & 6441.7 & 6804.1 & 12249.2 & 18602.1 & 131.4 & 67.8 & 34148 & 56.1 & 82.5 \\
\hline large & 90910.1 & 369518.0 & 251547.0 & 976670.8 & 1343.0 & 3325.9 & 10654 & 17.5 & 100 \\
\hline \multicolumn{10}{|c|}{ By technology level } \\
\hline High tech & 31054.5 & 347406.3 & 69194.1 & 764582.0 & 435.5 & 2554.8 & 7245 & 11.9 & 11.9 \\
\hline $\begin{array}{l}\text { High-medium } \\
\text { tech }\end{array}$ & 16401.1 & 99671.6 & 40201.7 & 288035.3 & 277.4 & 1398.4 & 26139 & 42.9 & 54.8 \\
\hline $\begin{array}{l}\text { Medium-low } \\
\text { tech }\end{array}$ & 22097.6 & 147556.9 & 67528.5 & 493035.6 & 280.4 & 1256.2 & 16807 & 27.6 & 82.4 \\
\hline Low tech & 18005.3 & 44117.0 & 43451.2 & 133776.5 & 390.1 & 784.4 & 10677 & 17.5 & 100 \\
\hline
\end{tabular}

Table 2 presents the descriptive statistics of the key characteristics of firms, i.e., the size and technology levels of firms. Value added, capital and labor are increased as firm size increases. For all the three variables, large discrepancies can be found between large and small firms. For example, the average value added of large firms is at least fifteen times larger than those of micro firms. The capital stock and labor of large firms are also larger than those of smaller firms. Although around $80 \%$ of our sample firms are categorized as small and medium firms, their output and employment shares are only around $20.4 \%$ and $14.9 \%$ of the total, respectively. As for the mean value of the variables by technology levels, although the number of firms in the high-technology level is smaller than in the other technology levels, the average output of the high-technology manufacturing sector is larger than that of the medium- and low-technology manufacturing sectors. The average input factors of firms in the high tech sector are on the whole larger than those in the other technology levels.

\section{Models}

\subsection{Productivity and technical change}

We assume that the firms' production function is best described as the following relationship between output, inputs and technology: 


$$
Y=f(X, t)
$$

where $Y$ is a scalar output, $X$ is a vector of inputs $(j=1, \cdots, J)$, and $t$ is the time trend variable representing technology. Taking total differential of equation (1) gives us the following equation:

$$
\dot{Y}=\sum_{j} \frac{f_{j} X_{j}}{Y} \dot{X}_{j}+\frac{f_{t}}{Y}=\sum \varepsilon_{j} \dot{X}_{j}+\frac{f_{t}}{Y}
$$

where the "dot" over a variable represents its growth rate. In equation (2) $f_{j}$ is the marginal product of the $j^{\text {th }}$ input, and $\varepsilon_{j}$ is the corresponding input elasticity.

We assume that the firms minimize cost and the input markets are competitive. Then, the relationship in equation (2) can be rewritten as:

$$
\dot{Y}-\sum_{j} S_{j} \dot{X}_{j}=\frac{f_{t}}{Y}+(R T S-1) \sum_{j} S_{j} \dot{X}_{j}
$$

where $S_{j}$ is the cost share of $j^{\text {th }}$ input, and $R T S=\sum_{j} \varepsilon_{j}$ denotes the returns to scale. The left-hand side of equation (3) is referred to as the Divisia index of TFP, expressed as:

$$
T F P_{D I V}=\dot{Y}-\sum_{j} S_{j} \dot{X}_{j}
$$

If price data is available to obtain the input cost shares, the above TFP growth measure can be calculated without an econometric estimation. Otherwise, econometric estimation of a production function is necessary. The main advantage of using a parametric approach over the non-parametric approach of the Divisia index is that, by avoiding the assumption of constant returns to scale, one can decompose TFP growth into technical change $\left(f_{t} / Y\right)$ and scale $\left((R T S-1) \sum_{j} S_{j} \dot{X}_{j}\right)$ components as indicated in equation (3), but at the cost of assuming a functional form for the input and output relationships.

\subsection{Time Trend (TT) and General Index (GI) Models}

In estimating our models, the following production model with panel data is assumed:

$$
y_{i t}=\beta_{0}+\mathbf{x}_{i t} \beta+u_{i t},
$$

where $y_{i t}$ is the $\log$ output of the producer $i \quad(i=1, \cdots, N)$ at time $t \quad(t=1, \cdots, T)$, $\mathbf{x}_{i t}$ is the corresponding vector of $J$ inputs and $\beta$ is a $J \times 1$ vector of unknown parameters to be estimated. In this study the composed error term, $u_{i t}$, is specified as: 


$$
u_{i t}=\mu_{i}+\lambda_{t}+v_{i t}
$$

where $\mu_{i}, \lambda_{t}$ and $v_{i t}$ represent firm-specific effects, time-specific effects and statistical noise, respectively. Random effects, which cannot be controlled for by the producers, are captured by the error term, $v_{i t}$, such as advantages or disadvantages in the location of the firm, access to labor, measurement errors in the dependent variables and left-out explanatory variables. We assume that this random error term is independently and identically normally distributed with zero mean and constant variance, $\sigma_{v}^{2}$. The firm-specific effect, $\mu_{i}$ is a factor representing producer efficiency, and the time-specific effect, $\lambda_{t}$, is a factor representing the exogenous rate of technical change (Heshmati, 2002). In order to avoid over-parameterization of the model the firm-specific effects, $\mu_{i}$, are replaced by industry-specific effects, $\eta_{d}$. Also, we assume a translog form of the production since it provides a good second-order approximation to a broad class of functions (Kneller and Andrew Stevens, 2003).

In the time trend model, the trend variable, $t$, is used as a regressor along with the input factor variables, $x$. The time-specific effect is specified as a linear function of a time trend. The basic time trend (TT1) model is written as:

$$
y_{i t}=\beta_{0}+\beta_{t} t+\frac{1}{2} \beta_{t t} t^{2}+\sum_{j} \beta_{j} x_{j i t}+\frac{1}{2} \sum_{j} \sum_{k} \beta_{j k} x_{j i t} x_{k i t}+\sum_{j} \beta_{j t} x_{j i t} t+\eta_{d}+v_{i t},
$$

where $t$ is a single time trend representing the exogenous rate of technical change. The $\eta_{d}$ is fixed industry-specific effects to be estimated. The TT1 model is assumed to satisfy the symmetry and convexity conditions.

In the general index model (GI1) of Baltagi and Griffin (1988), the trend variable $t$ is replaced by $A(t)$, where $A(t)(t=1, \cdots, T)$ are parameters to be estimated. The corresponding production function with the general index representation of technical change is given by

$$
y_{i t}=\beta_{0}+\sum_{j} \beta_{j} x_{j i t}+A(t)+\frac{1}{2} \sum_{j} \sum_{k} \beta_{j k} x_{j i t} x_{k i t}+\sum_{j} \beta_{j t} x_{j i t} A(t)+\eta_{d}+v_{i t},
$$

where the time trend and its square terms are replaced by $T-1$ fixed time-specific $A(t)$ effects. The GI1 model is also assumed to satisfy the symmetry and convexity conditions.

Since technical change is defined as the log derivative of output with respect to time, $(\partial y / \partial t)$, the rate of technical change (TC) in the TT1 model is given by:

$$
\mathrm{TC}_{\mathrm{TT} 1}=\beta_{t}+\beta_{t t} t+\sum_{j} \beta_{j t} x_{j} .
$$

And the corresponding rate in the GI1 model is given by: 


$$
\mathrm{TC}_{\mathrm{GI} I}=\{A(t)-A(t-1)\}\left\{1+\sum_{j} \beta_{j t} x_{j}\right\} .
$$

The TC expressed in equation (9) and (10) can be decomposed into components associated with pure time (neutral) effect and input variables (non-neutral) effects. These components in the TT1 model are $\beta_{t}+\beta_{t t} t$, and $\sum_{j} \beta_{j t} x_{j}$, respectively. In the GI model these components are $\{A(t)-A(t-1)\}$ and $\{A(t)-A(t-1)\} \sum_{j} \beta_{j t} x_{j}$, respectively. It is worth noting that there are some problems inherent in the nature of technical change in the TT1 model. First, the rate of technical change either increases $\left(\beta_{t t}>0\right)$ or decreases $\left(\beta_{t t}<0\right)$ linearly as a function of time. Second, with unbalanced panel data, it is not clear whether the trend variable, $t$, for a firm entering in period $\tau \quad(1<\tau<T)$ should start from $\tau$ or from 1. Third, in cases when the time span is relatively short or very long, a time trend model might not represent the exogenous technical change appropriately. Those problems are avoided in the GI1 model by estimating one parameter for each time period in $A(t)$.

Technical change can be biased towards a particular input. For an input $j$, bias $\left(B_{j}\right)$ in technical change is measured from $B_{j}=\partial S_{j} / \partial t$, where $S_{j}$ is the output elasticity of input $j$. A positive (negative) value of $B_{j}$ implies that technical change is relatively $j^{\text {th }}$ input using (saving). Zero value of $B_{j}$ indicates that technical change is not biased towards any particular input, i.e., technical change is neutral (Kumbhakar and Hjalmarsson, 1993). In the TT1 model, $B_{\mathrm{TT} 1, j}=\beta_{j t}$ which is a constant, and its sign is simply determined by the sign of $\beta_{j t}$. Hence, input bias in technical change derived from the TT1 model is firm- and time-invariant. In the GI1 model, however, input bias varies over time since $B_{\mathrm{GII}, j}=\beta_{j t}[A(t)-A(t-1)]$.

Like the input bias, scale bias in technical change can also be derived from $S B=\partial R T S / \partial t$, where $R T S=\sum_{j} \varepsilon_{j}$. In the TT1 model, the scale bias is given by $S B_{T T 1}=\sum_{j} \beta_{j t}$. Similarly, in the GI1 model, the scale bias is given by $S B_{G I 1}=[A(t)-A(t-1)] \sum_{j} \beta_{j t}$. The scale bias in the TT1 model is firm- and time-invariant, while in the GI1 model it is time-varying.

By using equations (3) and (9), TFP growth in the TT1 model is calculated as follows:

$$
\mathrm{TFP}_{\mathrm{TT} 1}=\mathrm{TC}_{\mathrm{TT} 1}+\left(\mathrm{RTS}_{\mathrm{TT} 1}-1\right) \sum_{j} \varepsilon_{j} \dot{x}_{j}
$$

where $\varepsilon_{j}=\partial y / \partial x_{j}=\beta_{j}+\sum_{k} \beta_{j k} x_{k}+\beta_{j t} t$, and $R T S=\sum_{j} \varepsilon_{j}$. If $R T S$ is greater than (equal to or less than) one, then there are increasing (constant or decreasing) returns to scale. Similarly, TFP growth in the GI1 model is as follows: 


$$
\mathrm{TFP}_{\mathrm{GI}}=\mathrm{TC}_{\mathrm{GI}}+\left(\mathrm{RTS}_{\mathrm{GI}}-1\right) \sum_{j} \varepsilon_{j} \dot{x}_{j}
$$

where $\varepsilon_{j}=\partial y / \partial x_{j}=\beta_{j}+\sum_{k} \beta_{j k} x_{k}+\beta_{j t} A(t)$, and $R T S=\sum_{j} \varepsilon_{j}$.

In the above two TFP growth measures, the difference between TFP growth and technical change is entirely attributed to RTS. If the production technology exhibits constant returns to scale, then TFP growth is identical to the rate of technical change.

\subsection{Extensions of Time Trend Model}

Although technical change in the TT1 model $\left(\mathrm{TC}_{\mathrm{TT} 1}\right)$ can be firm- and time-specific because input variables vary across firms, technical change is not firm-specific when the components related to input variables are all zero. This restrictive feature can be removed by extending the TT1 model in a much more flexible manner. For this study we use the model proposed by Cornwell et al. (1990) for these extensions. Using their model, but changing the context from technical efficiency to technical change, the TT2 model is specified as:

$$
y_{i t}=\beta_{d t}+\sum_{j} \beta_{j} x_{j i t}+\frac{1}{2} \sum_{j} \sum_{k} \beta_{j k} x_{j i t} x_{k i t}+\sum_{j} \beta_{j t} x_{j i t} t+v_{i t}
$$

where $\beta_{d t}$ are industry- and time-specific intercepts. By replacing $\beta_{d t}$ with a parametric function of time, the TT2 model considers the industry-specific effect in technical change. The model for the intercept $\left(\beta_{d t}\right)$ given in this study is specified as follows:

$$
\beta_{d t}=\eta_{1 d}+\eta_{2 d} t+1 / 2 \eta_{3 d} t^{2}
$$

where $\eta_{1 d}, \eta_{2 d}$ and $\eta_{3 d}$ are unknown parameters to be estimated. Hence, $\beta_{d t}$ is a quadratic function of time, varying across industries. The temporal pattern of $\beta_{d t}$ is flexible, and no further assumption is required. The rate of technical change in the TT2 model is thus expressed as follows:

$$
\mathrm{TC}_{\mathrm{TT} 2}=\eta_{2 d}+\eta_{3 d} t+\sum_{j} \beta_{j t} x_{j}
$$

Thus, $T C_{T T 2}$ is industry-specific, and it changes over time even when all input variables are zero. The pure component of technical change $\eta_{2 d}+\eta_{3 d} t$ is industry-specific. Industry-specific effects are not incorporated in the non-neutral technical change component, $\sum_{j} \beta_{j t} x_{j}$. In other words, the restriction imposed in this specification incorporates the temporal pattern across industries only with the pure technical change. The TFP growth of the TT2 model is as follows: 


$$
\mathrm{TFP}_{\mathrm{TT} 2}=\mathrm{TC}_{\mathrm{TT} 2}+\left(\mathrm{RTS}_{\mathrm{TT} 2}-1\right) \sum_{j} \varepsilon_{j} \dot{x}_{j}
$$

where $\operatorname{RTS}_{\mathrm{TT} 2}$ and $\varepsilon_{j}$ are returns to scale and elasticity of $j^{\text {th }}$ input, respectively.

Although the TT2 model is successful in making the pure component of technical change industry-specific, non-neutral technical change is still restrictive like the TT1 model. Now we consider another extension of the TT2 model, the TT3 model, which allows for estimation of industry-specific non-neutral rate of technical change. The production function of the TT3 model is as follows:

$$
y_{i t}=\beta_{d t}+\sum_{j} \beta_{j} x_{j i t}+\frac{1}{2} \sum_{j} \sum_{k} \beta_{j k} x_{j i t} x_{k i t}+\sum_{j} \zeta_{j d} x_{j i t} t+v_{i t}
$$

where $\beta_{d t}$ is the same as in the TT2 model, and $\zeta_{j d}(j=1, \cdots, J)$ are industry-specific unknown parameters to be estimated.

The rate of technical change obtained from the most general time trend model, TT3 model, is given by:

$$
\mathrm{TC}_{\mathrm{TT} 3}=\eta_{2 d}+\eta_{3 d} t+\sum_{j} \zeta_{j d} x_{j}
$$

TFP growth of the TT3 model is the same as in equation (16) except that the subscript TT2 is replaced with TT3.

It should be noted that input bias and scale bias in the TT2 model are the same as in the TT1 model, but in the TT3 model they are industry-specific. For input $j, B_{\mathrm{TT} 3, j}=\zeta_{j d}$ and its sign is simply determined by the sign of $\zeta_{j d}$. Scale bias is expressed as $S B_{T T 3}=\sum_{j} \eta_{j d}$.

\subsection{Extensions of General Index Model}

Under the specification of the GI1 model an implicit restriction is imposed on the temporal pattern of technical change across industries. This means that technical change varies over time, but it is the same across industries if components related with the input variables are all zero. This undesirable feature of invariant technical change across industries can be removed in such a way that the rate of technical change is industry- , time- and firm-specific. In this study we eliminate the restriction in two ways. In the first extended model, the GI2 model, by using Lee and Schmidt (1993) we make pure technical change industry-specific by specifying the production function as follows:

$$
y_{i t}=\eta_{d} A(t)+\sum_{j} \beta_{j} x_{j i t}+\frac{1}{2} \sum_{j} \sum_{k} \beta_{j k} x_{j i t} x_{k i t}+\sum_{j} \beta_{j t} x_{j i t} B_{j}(t),
$$


where $\eta_{d}$ are industry-specific parameters. In equation (19) $\eta_{d}, A(t)$ and $B_{j}(t)$ are unknown technology parameters to be estimated. Thus, the rate of technical change in the GI2 model is expressed as:

$$
\mathrm{TC}_{\mathrm{GI} 2}=\eta_{d}[A(t)-A(t-1)]+\sum_{j} \beta_{j t} x_{j}\left[B_{j}(t)-B_{j}(t-1)\right] .
$$

In equation (20), the industry specific effect is inherited in the pure component of technical change, $\eta_{d}[A(t)-A(t-1)]$. The non-neutral component of technical change is $\sum_{j} \beta_{j t} x_{j}\left[B_{j}(t)-B_{j}(t-1)\right]$. Note that industry-specific effects are not incorporated in the non-neutral component of technical change of the GI2 model. Also note that, unlike the extensions of time trend models, no functional form of technical change is assumed here. This is useful when the time span of panel data is narrow.

The GI2 model can be further extended when every $A(t)$ in equation (8) is replaced by industry-specific general indexes. The production function in the GI3 model is expressed as

$$
y_{i t}=\eta_{d} A(t)+\sum_{j} \beta_{j} x_{j i t}+\frac{1}{2} \sum_{j} \sum_{k} \beta_{j k} x_{j i t} x_{k i t}+\sum_{j} x_{j i t} \zeta_{j d} B_{j}(t)
$$

where $\zeta_{j d}$ are unknown parameters to be estimated. The rate of technical change in the GI3 model is written as:

$$
\mathrm{TC}_{\mathrm{GI} 3}=\eta_{d}[A(t)-A(t-1)]+\sum_{j} x_{j} \zeta_{j d}\left[B_{j}(t)-B_{j}(t-1)\right] .
$$

The GI3 model is much more flexible than the GI1 or GI2 models in that the industry-specific effects of the pure and non-neutral component of technical change are inherent in both.

Input bias and scale bias in the GI2 model are the same as those in the GI1 model. In the GI3 model, however, input bias and scale bias are both industry- and time-specific. These measures are expressed as:

$$
\begin{aligned}
& B_{\mathrm{TT} 3, j}=\zeta_{j d}\left[B_{j}(t)-B_{j}(t-1)\right] \\
& B S_{\mathrm{TT} 3}=\sum_{j} \zeta_{j d}\left[B_{j}(t)-B_{j}(t-1)\right] .
\end{aligned}
$$

\subsection{Non-parametric Approach}

This subsection provides a traditional measure of the TFP growth rate by the Solow residual approach. We begin with the following production function 


$$
Y_{i t}=A_{i t} F\left(K_{i t}, L_{i t}\right) \text {, }
$$

where $Y_{i t}, K_{i t}$ and $L_{i t}$ represent value added, capital stock and labor employment of firm $i$ in period $t . A_{i t}$ is a Hicks neutral technology index, which allows for shifts of the production function over time. By totally differentiating equation (24) and dividing it by $Y$, the following growth equation is obtained:

$$
\dot{Y} / Y=\varepsilon_{k} \dot{K} / K+\varepsilon_{l} \dot{L} / L+\dot{A} / A,
$$

where $\varepsilon_{k}$ and $\varepsilon_{l}$ represent elasticities of output with respect to capital and labor, respectively. By assuming constant returns to scale, $\varepsilon_{l}$ can be replaced with $1-\varepsilon_{k}$. Then, equation (25) can be expressed as follows:

$$
S R=\dot{Y} / Y-\left(1-\varepsilon_{k}\right) \dot{L} / L-\varepsilon_{k} \dot{K} / K,
$$

where the Solow residual $S R$ is equivalent to the estimate of TFP $(\dot{A} / A)$. Following the tradition in the literature, we specify the production function, $F(\cdot)$, as the Cobb-Douglas functional form.

\section{Empirical results}

\subsection{Estimation Methods}

In panel data literature, estimation of error component models has been developed in two main directions, the fixed effects (FE) and the random effects (RE) models. FE models assume that $\mu_{i}$ and $\lambda_{t}$ are fixed and correlated with the explanatory variables, and RE models assume that $\mu_{i}$ and $\lambda_{t}$ are random and not correlated with the explanatory variables. The choice between the FE and RE models affects various properties such as the efficiency, unbiasedness and consistency of estimated parameters. Hence, it is essential to choose the appropriate model to properly describe the nature of a data set. Here, we set up the following step for choosing the appropriate model: (i) in the TT1 and GI1 models, the firm-specific intercepts, $\eta_{i}$, are substituted by the industry-specific intercepts, $\eta_{d}$, (ii) the least-squares dummy variables (LSDV) method and the maximum likelihood estimation (MLE) method are employed to estimate the two models with FE and RE, respectively, (iii) the Hausman test is applied to choose the appropriate estimation method of the two alternative models, (iv) the chosen estimation method is assumed to be applicable to the extended models, (v) industry-specific intercepts, $\eta_{d}$, are included in the six model specifications instead of firm-specific intercepts. The assumption in (v) is necessarily imposed due to two reasons associated with the large data set: first, in terms of interpretation, it is not meaningful to observe fixed effects of individual firms because well-specified industry groups or firm sizes are a better target for the analysis; second, it is a quite time-consuming and challenging task for the specification and estimation if firm-specific intercepts are incorporated in 
nonlinear models such as the GI2 and GI3 models.

The results of the Hausman test signify that the FE model describes the nature of our data set better than the RE model. Hence, we use the FE model when estimating the six model specifications. The TT1, TT2, TT3 and GI1 models are linear and estimated using the LSDV method, while the GI2 and GI3 models are nonlinear and estimated by the MLE method. For each model, we assume that $v_{i t} \sim i . i . d N\left(0, \sigma_{v}^{2}\right)$ and $v_{i t}$ are independent of the explanatory variables. To capture the industry-specific features of TFP growth and technical change, 21 industry dummy variables are used following the industry classification rule of the OECD (2003).

\subsection{Specification Test and Model Selection}

The six model specifications (TT1, TT2, TT3, GI1, GI2 and GI3) outlined above are used to estimate the TFP growth and its decomposed components of Korean manufacturing firms for the period 1987-2007. In all the models the null hypothesis of constant returns to scale is rejected in favor of variable returns to scale at the $1 \%$ level of significance. The fit of the $R^{2}$ values is quite high, 0.83 , in all the models. The estimates of the parameters of the models are omitted in this paper to save space.

Although different assumptions on the behavior of technical change are inherent in each of the six models, it is essential to take into consideration the models that are appropriate for describing our data set. The obstacle to choosing the appropriate models is that our six models are not nested in a single super model. The TT1 model is nested in the TT2 and TT3, and the TT2 model is nested in the TT3. However, the GI1 model is not nested in the GI2 or GI3 models, but the GI2 model is nested in the GI3 model. Furthermore, the TT models are not nested in the GI models, and vice versa. We use the $\mathrm{J}$ test to choose the appropriate models among the non-nested models. For the nested models, the log-likelihood ratio test (LR test) is used to select appropriate models.

We report the best of the TT models, followed by the model selection among the GI models. We perform a LR test on the TT1 model against the TT2 and TT3 models. The LR test on these nested hypotheses (TT1 vs. TT2 and TT1 vs. TT3) rejects the TT1 model at the $1 \%$ level of significance. The result of the LR test on the TT2 and TT3 models also rejects the TT2 model in favor of the TT3 model. Thus, the test results give us conclusive evidence that the TT3 model is the best of the models with time trend model specifications.

The J test on the GI1 and the GI2 models shows that the GI1 model is not preferred to the GI2 model at the $1 \%$ level of significance. The J test result between the GI1 and the GI3 models shows that GI1 model is not preferred to the GI3 model at the 5\% level of significance. The LR test between the nested GI2 and the GI3 models shows that the GI3 model is chosen at the $1 \%$ level of significance. Hence, the model specification tests on the GI models indicate that the GI3 model is the most appropriate model.

We test the preferred TT model, TT3, against the preferred GI model, GI3. The J test on this model selection procedure is inconclusive. When discussing the TFP growth rate and its components, the results for both the TT3 and GI3 models are reported. The 
results for the TT1, TT2, GI1 and GI2 models can be obtained from the authors upon request.

\subsection{Input Elasticities and Returns to Scale}

The elasticities of output with respect to capital and labor inputs, $\varepsilon_{j}$, are calculated from $\varepsilon_{j}=\partial y / \partial x_{j}, j=l, k$. The returns to scale (RTS) are calculated from the sum of the input elasticities. These input elasticities and returns to scale vary across firms and over time.

Table 3. Mean elasticity and returns to scale based on TT3 and GI3 models

\begin{tabular}{|c|c|c|c|c|c|c|}
\hline & \multicolumn{3}{|c|}{ TT3 } & \multicolumn{3}{|c|}{ GI3 } \\
\hline & Capital & Labor & RTS & Capital & Labor & RTS \\
\hline \multicolumn{7}{|l|}{ Mean by size } \\
\hline Micro & 0.314 & 0.627 & 0.941 & 0.318 & 0.610 & 0.927 \\
\hline Small & 0.321 & 0.656 & 0.976 & 0.322 & 0.646 & 0.968 \\
\hline Medium & 0.344 & 0.661 & 1.004 & 0.336 & 0.658 & 0.993 \\
\hline Large & 0.382 & 0.657 & 1.039 & 0.373 & 0.662 & 1.035 \\
\hline \multicolumn{7}{|c|}{ Mean by technology level } \\
\hline High tech & 0.337 & 0.693 & 1.025 & 0.319 & 0.664 & 0.983 \\
\hline High-medium tech & 0.337 & 0.682 & 1.019 & 0.336 & 0.658 & 0.994 \\
\hline Medium-low tech & 0.348 & 0.644 & 0.993 & 0.346 & 0.651 & 0.997 \\
\hline Low tech & 0.361 & 0.602 & 0.963 & 0.347 & 0.647 & 0.994 \\
\hline Sample mean & 0.344 & 0.659 & 1.003 & 0.339 & 0.655 & 0.993 \\
\hline Sample StdDev & 0.062 & 0.081 & 0.050 & 0.056 & 0.060 & 0.039 \\
\hline
\end{tabular}

Table 3 presents the average elasticities with respect to firms' size and technology levels. The last two rows provide the overall sample mean and standard errors of input elasticities and RTS. The hypotheses of zero input elasticities are rejected for both inputs in each of the two models since the t-statistics based on the estimated elasticities and their standard errors are larger than 2 . The overall mean elasticities of output with respect to capital and labor slightly differ between the TT3 and GI3 models, but are of reasonable size. The capital elasticities of the TT3 and GI3 models are 0.344 and 0.339 , respectively. The labor elasticities of the TT3 and GI3 models are 0.659 and 0.655 , respectively. The fact that the labor elasticity is larger than the capital elasticity indicates that the increase of labor is more effective than the increase of capital for producing more output. Interestingly, the TT3 model exhibits increasing returns to scale, whereas the GI3 model exhibits decreasing returns to scale. The null hypotheses of constant returns to scale were rejected at the $1 \%$ level of significance for both models. This signifies that the Korean manufacturing firms in our sample are of sub-optimal size. It should be noted that, compared to industry level, a larger variation in each of the measure is found at the firm level.

The relationships between the size and elasticities are also presented in Table 3. A priori, one might expect that the degrees of capital and labor utilization will increase as the size 
of a firm increases, which is confirmed by the empirical results. Another interesting finding is that the returns to scale are positively correlated with firm size, and large firms exhibit increasing returns to scale while smaller firms exhibit decreasing returns to scale. This seems to reflect the feature of Korean industrial policies which was based on the state-led economic system, giving preference to large enterprises. This also signifies that the Korean economy needs industrial policies which foster small and medium enterprises (SMEs) to efficiently adjust their input factors so that they yield desirable higher levels of outputs.

The relationship between the technology level and elasticities are also presented in Table 3. Interestingly, compared with the low-tech manufacturing industry, the high-tech manufacturing industry has low capital elasticity and high labor elasticity for both the TT3 and GI3 models. In the high-tech industry, which requires creativity and inventiveness to bring into value added, it is essential for firms to employ well-educated and well-trained workers rather than to accumulate physical capital. Hence, the fact that the quality of labor force is quite important in the high-tech industry is reflected in the high labor elasticity. On the other hands, it is more effective for firms in the low-tech industry to replace workers with machinery because simple and monotonous work stages are more efficiently accomplished by machinery than by the labor force. Therefore, on average, the labor (capital) elasticity of high-tech industry is larger (smaller) than that of low-tech industry.

\subsection{Technical Change}

The development of the mean rate of technical change is shown in Figure 2. The overall mean rate of technical change is almost the same in the two models, and varies between 2.0 percent and 2.2 percent per year. All the TT models predict monotonic technical progress during the study period, from $0.8 \%$ in $1987-1988$ to $3.6 \%$ in $2006-2007$.

The estimated rates of technical change in the general index models, on the other hand, do not show a smooth uniform pattern over time. From Figure 2 we see fairly large variations in the rate of technical change during 1995-2000. Technical regress in this period coincides with the Monetary Crisis; as discussed in the Data section, large decreases in the utilization of inputs and output variables can be found from 1995, i.e. two years before the Monetary Crisis. However, any distinct technical regress during the Credit Crunch Crisis cannot be observed among the six models. The poor capital structure of Korean enterprises before the Monetary Crisis seems to be reflected in the technical regress which was preceded by the crisis, whereas the Credit Crunch Crisis caused by the high debt of households does not seem to be associated with technical change in the production of enterprises. 


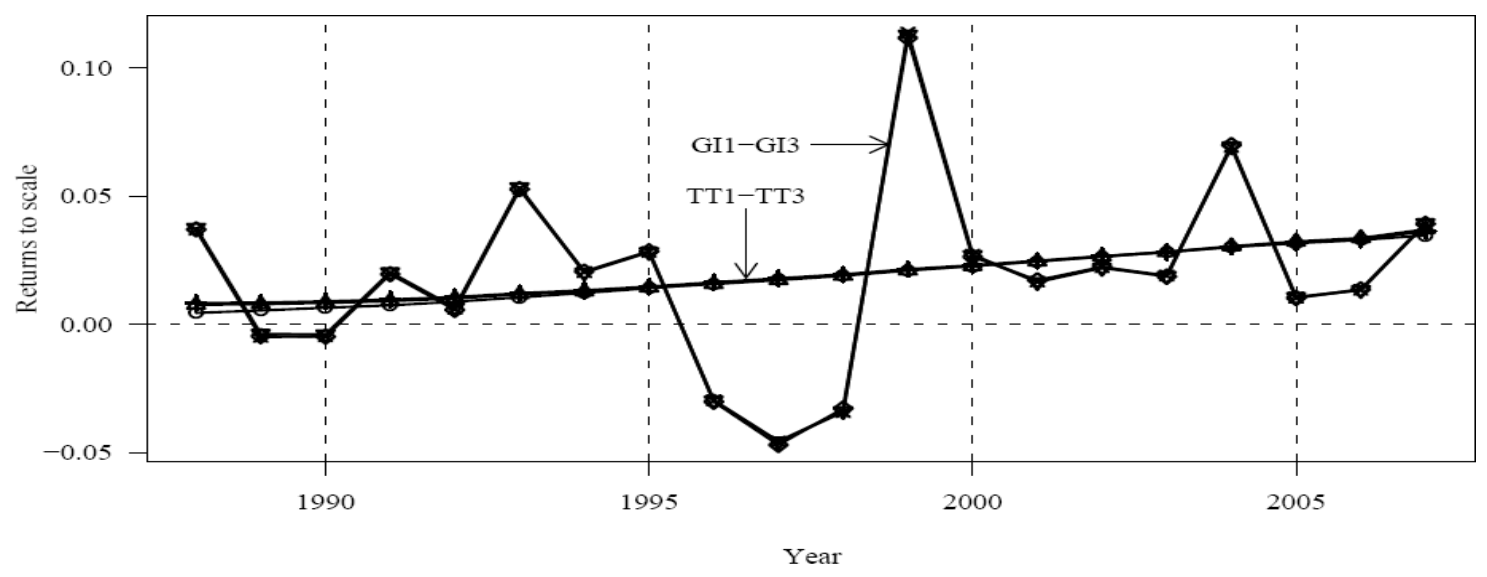

Figure 2. Mean rate of technical change based on TT1-TT3 and GI1-GI3 models

Table 4. Average technical change and its component based on TT3 and GI3 models

\begin{tabular}{|c|c|c|c|c|c|c|}
\hline & \multicolumn{3}{|c|}{ TT3 } & \multicolumn{3}{|c|}{ GI3 } \\
\hline & PUT & NNT & $\mathrm{TCH}$ & PUT & NNT & $\mathrm{TCH}$ \\
\hline \multicolumn{7}{|l|}{ Mean by size } \\
\hline Micro & 0.052 & -0.036 & 0.016 & 0.063 & -0.055 & 0.008 \\
\hline Small & 0.043 & -0.020 & 0.023 & 0.050 & -0.027 & 0.023 \\
\hline Medium & 0.035 & -0.013 & 0.021 & 0.039 & -0.021 & 0.019 \\
\hline Large & 0.028 & -0.004 & 0.024 & 0.034 & -0.013 & 0.021 \\
\hline \multicolumn{7}{|c|}{ Mean by technology level } \\
\hline High tech & 0.025 & 0.000 & 0.025 & 0.040 & -0.016 & 0.024 \\
\hline High-medium tech & 0.023 & 0.007 & 0.030 & 0.038 & -0.017 & 0.020 \\
\hline Medium-low tech & 0.037 & -0.016 & 0.021 & 0.036 & -0.017 & 0.019 \\
\hline Low tech & 0.034 & -0.023 & 0.011 & 0.026 & -0.004 & 0.022 \\
\hline Sample mean & 0.029 & -0.006 & 0.023 & 0.036 & -0.015 & 0.021 \\
\hline Sample StdDev & 0.029 & 0.022 & 0.017 & 0.179 & 0.174 & 0.056 \\
\hline
\end{tabular}

Note 1: PUT: pure component of technical change, NNT: non-neutral component of technical change, TCH: overall rate of technical change.

Note 2: Figures are calculated by the weighted mean, where weight is the value-added share of each firm.

The mean estimates of technical change by firm size classes are given in the upper panel of Table 4. Prior knowledge of the Korean industrial policies, which aim at fostering large firms, might make us expect large firms to exhibit a higher rate of technical progress. Interestingly, however, such expected tendencies cannot be found in our results. Instead of showing obvious relational patterns between the rate of technical change and size, the components show somewhat distinct patterns. That is, the pure component (non-neutral component) in the technical change of smaller firms is relatively larger (smaller) than that of larger firms. This indicates that smaller firms might not be good at coordinating input factors which are related to technical change, although their potential for achieving technical progress is relatively high. 
The average rates of technical change by technology levels are also provided in the lower panel of Table 4. The results of TT3 and GI3 show quite different patterns; first, the pure component of technical change in the TT3 (GI3) model is negatively (positively) related to the technology levels; second, the non-neutral component of technical change in the TT3 (GI3) model is positively (negatively) related to the technology levels; third, the average of technical change in the GI3 model is positively related to the technology levels, whereas the TT3 model shows no systematic association with respect to technology levels. These differences originate from the assumptions that we made concerning the flexible specification of technical change.

\subsection{Input Biases}

The overall mean input biases for all six models are presented in Table 5. Note that input and scale biases of the TT1 and TT2 models are constant. In general, the results differ across models, but are mostly of reasonable sizes. However, despite this difference, the patterns of factor using/saving biases are similar among the models, i.e., showing capital saving and labor using. Compared with the time trend models, the factor biases in the general index models are slightly larger. Another interesting fact is that the variations of input biases of the general index models are much larger than those of the time trend models.

The mean scale bias and the associated standard errors are presented in the last two columns of Table 5. The temporal variations of scale bias in all the models are rather small, around $0.2 \%$ per year. Like the input biases, variations of scale bias in the general index models are higher than those of the time trend models.

Table 5. Overall input bias and scale bias technical change

\begin{tabular}{ccccccccc}
\hline & \multicolumn{2}{c}{ Capital } & & \multicolumn{2}{c}{ Labor } & & \multicolumn{2}{c}{ Scale } \\
\cline { 2 - 3 } \cline { 7 - 8 } Model & Mean & S.D. & & Mean & S.D. & & Mean & S.D. \\
\hline TT1 & -0.66 & 0.00 & & 0.90 & 0.00 & & 0.24 & 0.00 \\
TT2 & -0.62 & 0.00 & & 0.85 & 0.00 & & 0.23 & 0.00 \\
TT3 & -0.61 & 0.34 & & 0.82 & 0.48 & & 0.22 & 0.33 \\
GI1 & -0.75 & 3.90 & & 0.92 & 4.32 & & 0.17 & 1.56 \\
GI2 & -0.78 & 3.93 & & 0.96 & 4.42 & & 0.19 & 1.52 \\
GI3 & -0.71 & 3.85 & & 0.91 & 4.26 & & 0.20 & 1.55 \\
\hline
\end{tabular}

\subsection{Total Factor Productivity Growth}

The development of the mean rate of TFP growth is shown in Figure 3. The overall mean rate of TFP growth is almost the same among the competing models, around $2.5 \%$ per year. The TT models show increasing trends in the rate of TFP growth over time, whilst the rate of TFP growth based on GI models does not show any systematic trends. The development patterns of the GI models mostly coincide with that of the Solow residual approach. Considering that the Solow residual approach is set as the reference 
for TFP growth measures, the GI models appear to suitably describe the actual rate of TFP growth. In addition, patterns of the rate of TFP growth in all the six models are very much similar to those of the technical change components, which signify that technical change is the main contributor to the TFP growth.

The average rates of TFP growth by firm size, technology level and industry are shown in Table 6. The mean rates of TFP growth differ among the six models, but are of reasonable size. In all the model specifications, the mean rate of TFP growth of large firms is found to be higher than that of small and medium enterprises (SMEs). This trend might come from the fact that the Korean industrial policies mainly focus on large firms rather than SMEs. A priori one would expect the average TFP growth of the high tech industry to be higher than that of other industries having a lower technology level, which is confirmed by the results. To sum up, large firms and high technology industries show a higher rate of TFP growth in the Korean manufacturing industry.

As regards the industry-level TFP growth, the mean rate of TFP growth differs among the models, but is of reasonable size except for the pharmaceutical industries. The pharmaceutical industry shows quite high growth rate in the TT3 model, whereas it shows a negative growth in the TT2 model. This result is related, not to any data problem but to the model specification and its suitability for studying heterogeneous industrial sectors. It is a common problem in applied economics research to obtain contradicting results when employing competing models for measuring TFP growth. The interesting fact deduced from Table 5 is that the radio, television and communication equipment branch (TELCOM) shows an annual growth rate of more than 3\%, regardless of model specifications. It seems to reflect the dramatic expansion of the branch in Korea in the last decade. The shipbuilding branch (SHIPBT), which is one of the strong branches of the Korean economy, also shows a high rate of TFP growth for all the six models. The chemical sector, which was chosen as one of the main industries in the Five-Year Economic Development Plans during the 1960s and 1970 s in Korea, ${ }^{1}$ is among the unexpected results. It does not show such a high TFP growth, only around $2 \%$, in all the models.

\footnotetext{
${ }^{1}$ The Five-Year Economic Development Plans were set up by the Korean government in order to select and develop the industrial sectors, especially heavy industries, for the period 1962-1981.
} 


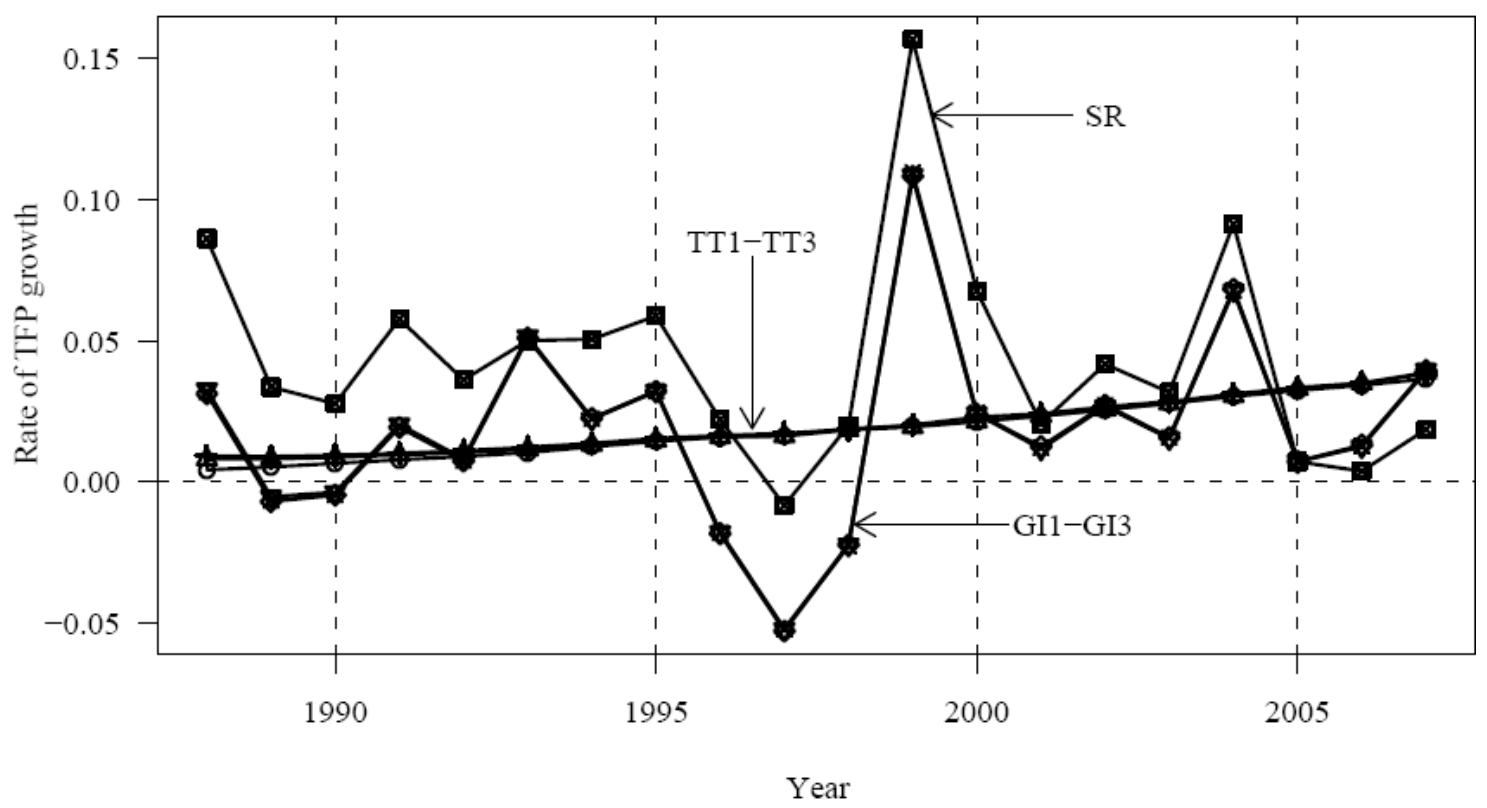

Figure 3. Average TFP growth by year based on Solow Residual (SR), TT1-TT3 and GI1-GI3 models 
Table 6. Average rate of TFP growth based on TT1-TT3 and GI1-GI3 models (\%)

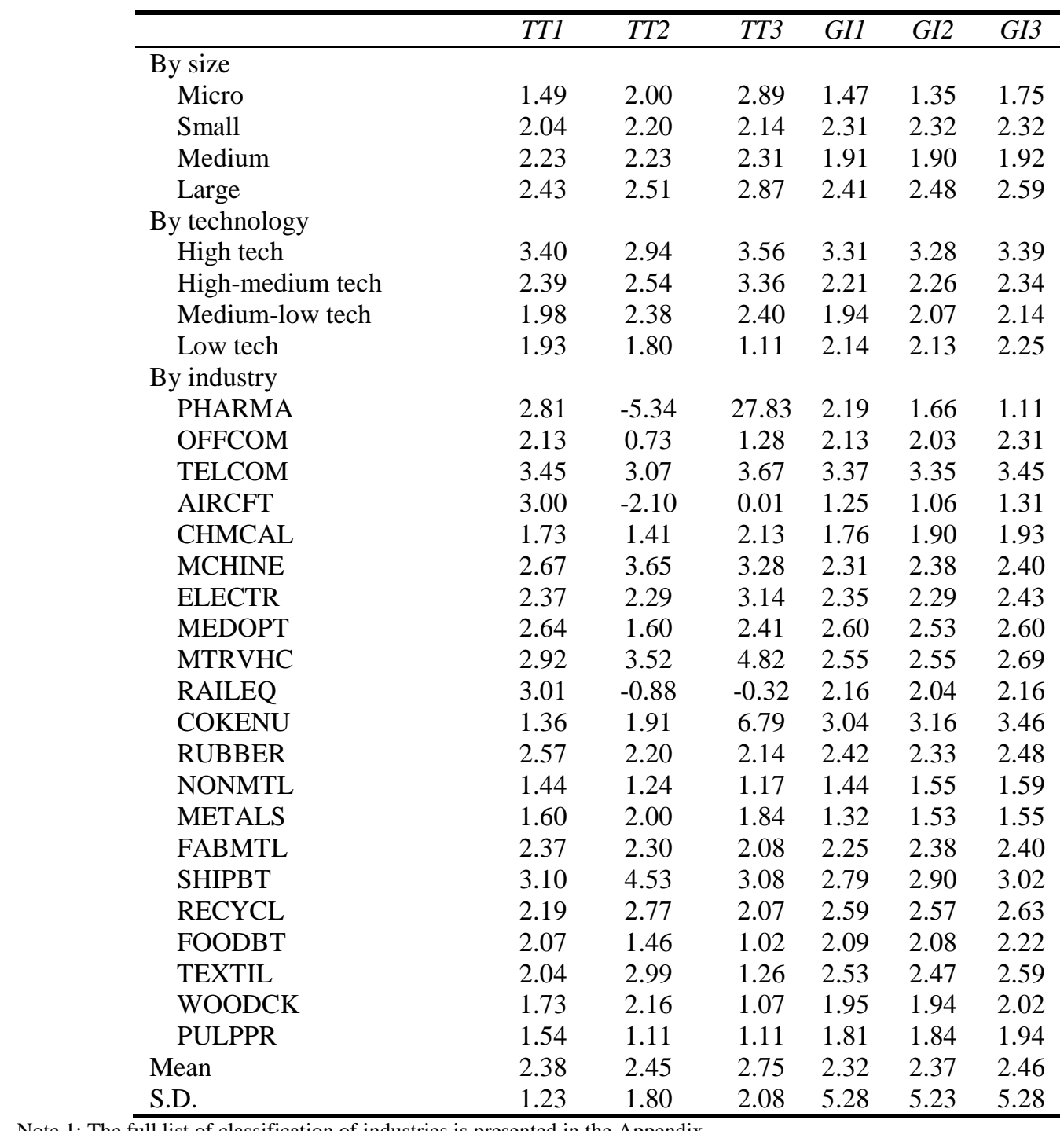

Note 1: The full list of classification of industries is presented in the Appendix.

Note 2: Rates of TFP growth are calculated by weighted mean, where the weight is the value added of each firm.

The Spearman rank correlation of TFP growth rates for the six models are given in Table 7. All the pairs of cases are positively correlated. The correlation coefficients of the models are reasonable, highly statistically significant and within the range of 0.13-0.99. The highest correlation coefficient among the models is found between the GI1 and the GI3 models (0.998) and the lowest surprisingly between the two accepted TT3 and the GI3 models (0.135). The low correlation is attributed to the differences in the two modeling approaches' ability to capture the dynamics of technical change. As is shown, the time trend models, in spite of being able to predict the average rates reasonably well, are not able to capture the erratic temporal patterns of technical change. 
Table 7. Correlations between the TFP mean values of the models; Spearman correlation coefficients

\begin{tabular}{ccccccc}
\hline & $T T 1$ & $T T 2$ & $T T 3$ & $G I 1$ & $G I 2$ & $G I 3$ \\
\hline TT1 & 1.000 & & & & & \\
TT2 & $0.673^{\mathrm{a}}$ & 1.000 & & & & \\
TT3 & $0.593^{\mathrm{a}}$ & $0.869^{\mathrm{a}}$ & 1.000 & & & \\
GI1 & $0.189^{\mathrm{a}}$ & $0.147^{\mathrm{a}}$ & $0.136^{\mathrm{a}}$ & 1.000 & & \\
GI2 & $0.190^{\mathrm{a}}$ & $0.154^{\mathrm{a}}$ & $0.140^{\mathrm{a}}$ & $0.993^{\mathrm{a}}$ & 1.000 & \\
GI3 & $0.186^{\mathrm{a}}$ & $0.149^{\mathrm{a}}$ & $0.135^{\mathrm{a}}$ & $0.998^{\mathrm{a}}$ & $0.992^{\mathrm{a}}$ & 1.000 \\
\hline
\end{tabular}

Note: The superscript ${ }^{\mathrm{a}}$ indicates statistical significance at less than $1 \%$ level of significance.

\subsection{Second Stage Regression Analysis of TFP growth}

In order to find the intensities of the individual determinants of TFP growth, we conduct a second stage regression analysis. Since we have six sets of firm-level TFP growth rates, we use each TFP growth rate as a dependent variable in this second stage regression analysis. Independent variables considered as determinants of TFP growth are as follows: capital intensity, market competition condition, wage growth, age and patenting activities of firms, and dummy for conglomerate membership.

Capital intensity is considered as a measure of firm-specific knowledge embodied in the machinery and equipment in production. Hence, firms with a higher level of capital intensity are expected to have high asset specificity and more variability in capital utilization. The possibility of increase in the rental cost of unused capital makes firms use their production resources efficiently (Jung, 1991). However, empirical studies show somewhat mixed results. For example, Lim (1980) and Sheehan (1997) give support to the positive relationship between the level of capital intensity and the firms' performance, whereas Ferrier et al. (1998) and Mahadevan and Kalirajan (2000) report a negative effect of capital intensity on production. In this paper, the ratio of capital to the number of employees is defined as the capital intensity. The growth rate of capital intensity is used as one of the determinants of TFP growth. We have named it CAPINT.

Regarding the relationship between the performance and the competitive condition of a market, two different points of view exist. Neoclassical economists support a positive relationship between the two measures, arguing that the elimination of slacks promotes the performance. Schumpeterians assert a negative relationship, pointing out that monopoly rents induce entrepreneurs to invest in R\&D activities and thus promote the dynamic performance. Empirical studies also provide different results. Nickell (1996), Aghion et al. (2001) and Boone (2001) find some support for the view that competition improves performance, whereas Dasgupta and Stiglitz (1980) substantiate the Schumpeterian view. We measure the competition by using the Herfindahl index, $\sum_{i} s_{i}^{2}$ where $s_{i}$ is the market share of the $i^{\text {th }}$ firm. We have named it MKTCOM. Note that the Herfindahl index close to unity indicates a less competitive market condition.

The relationship between wages and TFP growth also needs to be examined. Since TFP growth is positively associated with the labor productivity, firms exhibiting a high level of TFP growth also tend to yield a high labor productivity growth. Considering that 
firms' labor demand is mainly determined by labor's productivity, it is likely that wages will increase if the employee is more productive. Hence, we expect a positive relationship between the wage growth and the growth of TFP. In order to examine this relationship, the growth rates of total compensation of each firm are calculated and labeled as WGGRTH.

A fourth factor related to TFP growth is the age of the firm. The two main theoretical strands concerning the age and productivity are quite different: positive effect vs. negative effect. The theory on the positive impact of age suggests that older firms, having more experience and enjoying the benefits of learning, tend to yield a better performance than newer firms (Stinchcombe, 1965). The other strand of theoretical research shows that older firms, not being prone to change and having bureaucratic ossification that goes with age, lack the flexibility to rapidly adjust to changing circumstances (Marshall, 1920). In line with the aforementioned conflicting theoretical strands, empirical studies also show quite different results. Two such cases with conflicting results are Majumdar (1997) and Kutsuna et al. (2002). Here, we calculate the age of the firm by the number of years of operation, and label it as AGE.

As already found in the relationship between technical change and productivity growth, technical change is highly correlated with productivity growth. The rate of technical change is mainly driven by the creation and maintenance of intellectual property rights, which are commonly represented by patenting activities (Basmann et al., 2007). Hence, patenting activities, the outcome of which affects the innovative activities of firms (Crépon et al., 1998), are expected to be positively related with total factor productivity growth. We assume here that patenting activity is captured by the number of patents published and refer to it as PATENT. The patent data is from the Korea Industrial Property Rights Information Server (KIPIRS). The number of patent publications for each firm is computed and merged with the administrative register data.

Strongly state-led industrial policies have matured chaebols which are Korean-specific conglomerates. The chaebols are family-controlled, diversified business groups, and have dominated the Korean economy for several decades. Many applied studies on the chaebols and productivity growth, such as Lim (1999), suggest that chaebol firms have a higher productivity growth than non-chaebol firms. They argue that the chaebol leadership has been central to Korean economic growth and technological advancement. Kim et al (2006) find that chaebol firms have a better access to capital and are able to adjust their capital structure to optimal level much faster than their non-chaebol counterparts. However, more recent studies, such as Kim (2006), argue that the chaebols' productivity level was much lower than that of non-chaebols for the period 1991-1998. To find rigorous evidence from the firm-level data for a long period, we examine the effect of chaebol membership on the productivity growth. Following the definition of chaebols by Kim (2006), we have created a dummy variable for this category and named it CHAEBOL.

When attempting to isolate the relationships of the six firm-specific characteristic variables above and TFP growth, it is essential to control for other independent variables that are likely to affect the TFP growth. This is also important in dealing with the heterogeneity of firms in our sample. Among the various firm-specific attributes that are shared by firms, we have chosen the following control variables: KOSPI dummy, 
year dummy and industry dummy. ${ }^{2}$ The KOSPI dummy is included in order to isolate the effect of chaebols since most chaebol branches are listed companies and part of the KOSPI index.

To model the second stage regression analysis, each of the six TFP growth measures is used as a dependent variable along with the six independent variables above. The fixed-effects type of regression is used to find the parameter estimates. Table 8 presents the parameter estimates of the second stage regression. Overall, the estimation results show a good fit.

In all six models, the coefficient of the CAPINT is negative and statistically significant at the $1 \%$ level of significance, suggesting that high capital intensity decreases TFP growth. This result might come from the fact that most of the excess capital stock became obsolete during the economic recession in the aftermath of the 1997 Monetary Crisis.

Regarding the market competition, the signs as well as significant levels differ across different model specifications. Results of the TT models support the hypothesis that higher competition decreases TFP growth at the $1 \%$ level of significance, whereas results of the GI models yield inconclusive and insignificant results for this relation. From these results it can be concluded that the low competitive conditions for Korean manufacturing industries have increased their productivity. In other words, an oligopolistic market condition has increased the performance of the Korean manufacturing industry. Considering that the Korean economy is mainly led by several giant conglomerates, chaebols, this is quite reasonable. Despite such findings, however, we cannot say that this oligopolistic market condition will continuously yield high productivity growth. This suggests that the results should be interpreted with caution for future industrial planning.

The estimated coefficients of WGGRTH are of different sizes and signs. In the TT models, we obtain the expected (positive) signs, supporting our hypothesis. In the GI models, however, we obtain unexpected (negative) signs, which in the GI3 is statistically significant at the $1 \%$ level of significance. The large decline in wages during the Monetary Crisis might be reflected in the GI models. With these conflicting results, unfortunately, we fail, in line with our hypothesis, to establish a relationship between wage growth and TFP growth.

We observe a positive and statistically significant coefficient for the AGE variable among all the model specifications. This helps us to choose one of the strands which describe the positive relationship between the age of firms and their performance. Based on this, we can say that old firms enjoy the benefits of accumulated skills, management and technology in achieving a higher productivity growth. This implies that industrial policies for sustaining old enterprises need to be developed to increase the performance of the manufacturing industries.

We can also observe a positive and statistically significant coefficient for the PATENT

\footnotetext{
${ }^{2}$ KOSPI is the Korea Composite Stock Price Index, which is the index of all common stocks traded on the Stock Market Division.
} 
variable among the model specifications. These results coincide with our expectation that growth in patenting activities increases the rate of TFP growth by stimulating innovativeness of firms. Hence, future industrial policy must be oriented towards protecting intellectual property rights, especially for fostering technical progress. This policy implication is in line with Kang et al. (2008), who argue that the policy for credit guarantees for technology needs to be revised, since the allocation of credit guarantees to technology firms is not consistent with the policy program objectives.

All the estimated coefficients of the CHAEBOL variables are positive, signifying that the TFP growth of chaebols is superior to that of non-chaebol firms. The statistically significant levels of estimates are different among the six models. In the TT models it is statically significant at less than the 5\% level of significance, whereas in the GI models none of our specifications yield significant results. Like the results of the market condition, however, these results need to be interpreted with caution in any future policy making.

Table 8. Results of second stage regression analysis of determinants of TFP growth $(n=53,618)$

\begin{tabular}{|c|c|c|c|c|c|c|c|}
\hline & $\begin{array}{c}\text { Expected } \\
\text { sign }\end{array}$ & TT1 & TT2 & TT3 & GI1 & GI2 & GI3 \\
\hline Intercept & & $2.64 \mathrm{e}-03$ & $-2.10 \mathrm{e}-01^{\mathrm{a}}$ & $2.62 \mathrm{e}-01^{\mathrm{a}}$ & $3.02 \mathrm{e}-02$ & $2.94 \mathrm{e}-02$ & $2.32 \mathrm{e}-02$ \\
\hline CAPINT & $(+/-)$ & $-3.86 \mathrm{e}-03^{\mathrm{a}}$ & $-3.53 e-03^{\mathrm{a}}$ & $-3.74 \mathrm{e}-03^{\mathrm{a}}$ & $-2.70 \mathrm{e}-03^{\mathrm{a}}$ & $-3.16 \mathrm{e}-03^{\mathrm{a}}$ & $-2.86 \mathrm{e}-03^{\mathrm{a}}$ \\
\hline MKTCOM & $(+/-)$ & $1.04 \mathrm{e}-02^{\mathrm{a}}$ & $2.67 \mathrm{e}-02^{\mathrm{a}}$ & $1.36 \mathrm{e}-02^{\mathrm{a}}$ & $-5.90 e-03$ & $-4.69 \mathrm{e}-03$ & $6.27 \mathrm{e}-05$ \\
\hline WGGRTH & $(+)$ & $1.58 \mathrm{e}-04$ & $4.26 \mathrm{e}-04^{\mathrm{b}}$ & $1.11 \mathrm{e}-03^{\mathrm{a}}$ & $-6.97 e-04$ & $-7.85 e-04$ & $-1.35 \mathrm{e}-03^{\mathrm{a}}$ \\
\hline AGE & $(+/-)$ & $3.63 \mathrm{e}-05^{\mathrm{a}}$ & $5.30 \mathrm{e}-05^{\mathrm{a}}$ & $3.71 \mathrm{e}-05^{\mathrm{a}}$ & $3.44 \mathrm{e}-05^{\mathrm{c}}$ & $3.82 \mathrm{e}-05^{\mathrm{b}}$ & $5.87 \mathrm{e}-05^{\mathrm{a}}$ \\
\hline PATENT & $(+)$ & $4.71 \mathrm{e}-06^{\mathrm{a}}$ & $6.67 \mathrm{e}-06^{\mathrm{a}}$ & $1.02 \mathrm{e}-05^{\mathrm{a}}$ & $1.82 \mathrm{e}-05^{\mathrm{a}}$ & $2.01 \mathrm{e}-05^{\mathrm{a}}$ & $1.92 \mathrm{e}-05^{\mathrm{a}}$ \\
\hline CHAEBOL & $(+/-)$ & $4.45 \mathrm{e}-04^{\mathrm{a}}$ & $4.19 \mathrm{e}-04^{\mathrm{b}}$ & $1.06 \mathrm{e}-03^{\mathrm{a}}$ & $4.67 \mathrm{e}-04$ & $5.16 \mathrm{e}-04$ & $7.15 \mathrm{e}-04$ \\
\hline $\mathrm{R}^{2}$ & & 0.574 & 0.444 & 0.322 & 0.420 & 0.425 & 0.414 \\
\hline $\operatorname{adj} R^{2}$ & & 0.573 & 0.444 & 0.322 & 0.419 & 0.425 & 0.414 \\
\hline
\end{tabular}

Note: The superscripts a, b and c indicate statistical significance at less than 1, 1-5 and 5-10 levels.

\section{Conclusion}

In this paper, we consider estimation of total factor productivity growth, technical change and its related measures using firm level panel data for the Korean manufacturing industry for the period 1987-2007. In order to obtain more robust results, we employ competing flexible model specifications, along with the non-parametric Solow residuals approach serving as a benchmark. In doing so, we use six different model specifications: the time trend model, the general index model, and their extensions. The extensions of the two basic models allow for incorporation of firm-specific heterogeneity in technical change and its underlying components. These models are employed to analyze firm-level manufacturing data. Along with the examination of TFP growth, we also attempt to identify the determinants of TFP growth by means of second stage regression analysis and quantify the individual factor impacts.

The results of the model selection tests are mixed. Among the TT models, the most 
general TT3 model is found to be the appropriate model specification. Among the GI models, the most general GI3 model is chosen as the appropriate model. In these two model specifications the firm-specific rate of technical change is set up in the most flexible manner. Using test statistics, we fail to choose the best model between the non-nested TT3 and GI3 models. However, examination of the patterns of TFP growth and technical change enables us to choose the GI3 model as the best model among our six model specifications.

Based on the empirical results, it is possible to make several concluding points. First, the results of the returns to scale provide different perspectives: increasing returns to scale in the TT3 model and decreasing returns to scale in the GI3 model. Second, labor elasticity is larger than capital elasticity in all the model cases. Third, similarities exist in the overall rate of technical change among the two groups of models, whereas TT models and GI models yield different patterns during the study period. Fourth, all of the TT models yield an increasing trend in the rate of technical change, while all the GI models show a negative rate of technical change for 1995-1998. The results in the GI models signify that the productivity started to degenerate two years before the Monetary Crisis. Fifth, neither the difference in firm size nor the difference in technology levels shows a distinct difference in the temporal patterns of technical change, whereas they are related to the components of technical change. Sixth, evidence of model dependency exists in the input and scale biases, but its discrepancy is of reasonable size. Seventh, the overall average rate of TFP growth is almost the same for all the models. Eighth, temporal patterns of TFP growth of the TT models exhibit an increasing trend, whereas those of the GI models almost coincide with the Solow residual approach without showing systematic smooth patterns. Ninth, the result of the second regression indicates that i) the capital intensity growth and the competitive market condition are negatively related to the rate of TFP growth, ii) the age and patenting activities of the firm positively affect its TFP growth, and that iii) the TFP growth rate of chaebols is higher than that of non-chaebol firms.

To our knowledge, this paper is the first attempt to analyze the TFP growth, based on firm-level data, of the Korean manufacturing industry by employing competing parametric flexible model formulations and rigorous sensitivity analysis. We believe that this study provides information about TFP growth that can be useful in industrial policy decision makings. Studies using other forms of model specification, such as Stevenson (1980), are also believed to contribute to strengthening the robustness of the results. 


\section{References}

Aghion, P., Harris, C., Howitt, P., Vickers, J., 2001. Competition, imitation and growth with step-by-step innovation. Review of Economics Studies 68(3), 467-492.

Baltagi, B. H., Griffin, J. M., 1988. A general index of technical change. The Journal of Political Economy 96(1), 20-41.

Basmann, R. L., McAleer, M., Slottje, D., 2007. Patent activity and technical change. Journal of Econometrics 139(2), 355-375.

Boone, J., 2001. Intensity of competition and the incentive to innovate. International Journal of Industrial Organization 19 (5), 705-726.

Cornwell, C., Schmidt, P., Sickles, R. C., 1990. Production frontiers with cross-sectional and time-series variation in efficiency levels. Journal of Econometrics 46(1-2), 185-200.

Crépon, B., Duguet, E., Mairesse, J., 1998. Research, innovation, and productivity: An econometric analysis at the firm level. Working Paper 6696, National Bureau of Economic Research.

Dasgupta, P., Stiglitz, J., 1980. Industrial structure and the nature of innovative activity. Economic Journal 90(358), 266-293.

Diewert, W. E., 1981. The theory of total factor productivity measurement in regulated industries. Academic Press, New York.

Dopfer, K., Foster, J., Potts, J., 2004. Micro-meso-macro. Journal of Evolutionary Economics 14 (3), 263-279.

Ferrier, G. D., Klinedinst, M., Linvill, C. B., 1998. Static and dynamic productivity among Yugoslav enterprises: Components and correlates. Journal of Comparative Economics 26(4), 805-821.

Fu, F.-C., Vijverberg, C.-P., Chen, Y.-S., 2008. Productivity and efficiency of state-owned enterprises in china. Journal of Productivity Analysis 29 (3), 249-259.

Heshmati, A., 2002. Productivity measurement in Swedish departments of gynecology and obstetrics. Structural Change and Economic Dynamics 13(3), 315-336.

Jung, Y., 1991. Multinationality and profitability. Journal of Business Research 23(2), 179-187.

Kang J.W., A. Heshmati and G.G. Choi, 2008. The Effects of Credit Guarantees on Survival and Performance of SMEs in Korea, Small Business Economics 31(4), 445-462.

Kim, E., 2006. The impact of family ownership and capital structures on productivity 
performance of Korean manufacturing firms: Corporate governance and the "Chaebol problem". Journal of the Japanese and International Economies 20(2), 209-233.

Kim H-S., A. Heshmati and D. Aoun, 2006. Dynamics of Capital Structure: The Case of Korean Listed Manufacturing Companies, Asian Economic Journal 20(3), 275-303.

Kneller, R., Andrew Stevens, P., 2003. The specification of the aggregate production function in the presence of inefficiency. Economics Letters 81(2), 223-226.

Kumbhakar, S. C., Hjalmarsson, L., 1993. Technical Efficiency and Technical Progress in Swedish Dairy Farms. Oxford University Press, Oxford, pp. 256-270.

Kutsuna, K., Okamura, H., Cowling, M., 2002. Ownership structure pre- and post-IPOs and the operating performance of JASDAQ companies. Pacific-Basin Finance Journal 10(2), 163-181.

Lee, Y. H., Schmidt, P., 1993. The measurement of productive efficiency: techniques and applications. Oxford University Press, Oxford, pp. 237-255

Lim, D., 1980. Tax incentives and resource utilization in a small, less developed country. Australian Economic Papers 19(34), 22-30.

Lim, Y., 1999. Technology and Productivity: The Korean Way of Learning and Catching Up. MIT Press, Cambridge.

Little, R., An, H., 2004. Robust likelihood-based analysis of multivariate data with missing values. Statistica Sinica 14(3), 949-968.

Mahadevan, R., Kalirajan, K., 2000. Singapore's manufacturing sector's TFP growth: A decomposition analysis. Journal of Comparative Economics 28(4), 828-839.

Majumdar, S. K., 1997. The impact of size and age on firm-level performance: Some evidence from India. Review of Industrial Organization 12(2), 231-241.

Marshall, A., 1920. Principles of Economics, 8th Edition. Macmillan, London.

Nickell, S., 1996. Competition and corporate performance. Journal of Political Economy 104, 724-746

OECD, 2003. The Source of Economic Growth in OECD Countries. OECD, Paris, France.

Sheehan, M., 1997. The evolution of technical efficiency in the Northern Ireland manufacturing sector. Scottish Journal of Political Economy 44(1), 59-81.

Stevenson, R. E., 1980. Likelihood functions for generalized stochastic frontier estimation. Journal of Econometrics 13(1), 57-66.

Stinchcombe, A. L., 1965. Social Structure and Organizations. Handbook of Organizations. Rand McNally, Chicago. 


\section{Appendix}

Table 9. Classification of industries

\begin{tabular}{|c|c|c|}
\hline Acronym & Explanation & $S I C$ \\
\hline PHARMA & Pharmaceuticals & 2423 \\
\hline OFFCOM & Office accounting and computing equipment & 30 \\
\hline TELCOM & Radio television and communication equipment & 32 \\
\hline AIRCFT & Aircraft and space craft & 353 \\
\hline CHMCAL & Chemicals w/o pharmaceuticals & 24 exc. 2423 \\
\hline MCHINE & Machinery and equipment & 29 \\
\hline ELECTR & Electrical equipment and apparatus & 31 \\
\hline MEDOPT & Medical precision and optical instruments & 33 \\
\hline MTRVHC & Motor vehicles trailers and semi-trailers & 34 \\
\hline RAILEQ & Railroad equipment and transport equipment & $352-359$ \\
\hline COKENU & Coke refined petroleum product and nuclear fuel & 23 \\
\hline RUBBER & Rubber and plastic products & 25 \\
\hline NONMTL & Other non-metallic mineral products & 26 \\
\hline METALS & Basic metals & 27 \\
\hline FABMTL & Fabricated metal products except machinery and equipment & 28 \\
\hline SHIPBT & Building and repairing of ships and boats & 351 \\
\hline RECYCL & Manufacturing n.e.c. recycling & $36-37$ \\
\hline FOODBT & Food products, beverage, and tobacco & $15-16$ \\
\hline TEXTIL & Textile products leather and footwear & $17-19$ \\
\hline WOODCK & Wood and products of wood and cork & 20 \\
\hline PULPPR & Pulp paper products printing and publishing & $21-22$ \\
\hline
\end{tabular}

Note: SNI is the standard industrial code.

i The gross output is not considered as an alternative measure to value added since quantities of material inputs are not available in the KIS-VALUE data. 